\title{
Development of Superconducting Tunnel Junction Far-Infrared Photon Detector for Cosmic Background Neutrino Decay Search - COBAND Experiment
}

S. H. Kim ${ }^{a},{ }^{*},[1]$, Y. Takeuchi ${ }^{a,{ }^{*}}$, T. lidaa ${ }^{a}$, K. Takemasa ${ }^{a}$, K. Nagata ${ }^{a}$, C. Asano ${ }^{a}$, R. Wakasa ${ }^{a}$, S. Yagi ${ }^{a}$, A. Kasajima ${ }^{a}$, H. Ikeda ${ }^{b}$, T. Wada $^{b}$, K. Nagase ${ }^{\text {, }}$,

S. Matsuurac ${ }^{\text {, }}$ M. Hazumi ${ }^{\text {d }}$, Y. Arai ${ }^{\text {d }}$, I. Kurachi ${ }^{\text {d }}$, T. Yoshida ${ }^{e},{ }^{*}$, M. Sakai ${ }^{e}$, T. Nakamurae ${ }^{e}$, W. Nishimura ${ }^{e}$, K. Kiuchi ${ }^{f}$, S. Mima ${ }^{f}$, Y. Kato ${ }^{g}$, M. Ohkubo ${ }^{\text {, }}$,

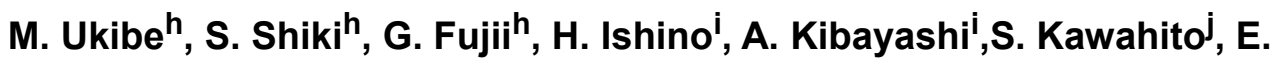
Rambergk $^{k}$, P. Rubinov", D. Sergatskov' ${ }^{k}$ and S.B. Kim ${ }^{\text {l,* }}$

a) University of Tsukuba, Tsukuba, Ibaraki, 305-8571 Japan

b) JAXA ISAS, Sagamihara, Kanagawa,252-5210, Japan

c) Kwansei Gakuin University, Hyogo 669-1337, Japan

d) KEK, Ibaraki 305-0801, Japan

e) University of Fukui, Fukui 910-8507, Japan

f) RIKEN, Saitama 351-0198, Japan

g) Kindai University, Osaka 577-8502, Japan

h) AIST, Ibaraki 305-8560, Japan

I) Okayama University, Okayama 700-8530, Japan

j) Shizuoka University, Shizuoka 422-8529, Japan

k) Fermi National Accelerator Laboratory, Illinois, 60510, US

l) Seoul National University, Seoul, 151-742, Korea

*) Tomonaga Center for the Histoty of the Universe at University of Tsukuba

\begin{abstract}
We present the status of development of the Superconducting Tunnel Junction (STJ) detector for the COsmic BAckground Neutrino Decay search (COBAND) experiment. The signal of the cosmic background neutrino decay is identified as a sharp cutoff at high energy end in a far-infrared region ranging from $15 \mathrm{meV}$ to $30 \mathrm{meV}$ in the energy spectrum of the photons from the space. The COBAND experiment will be done as rocket and satellite experiments in order to detect the far-infrared photons from the space. For the photon detector of the COBAND experiment, we are developing the STJ detectors. We will use a combination of the diffraction grating and the array of $\mathrm{Nb} / \mathrm{Al}-\mathrm{STJ}$ pixels with cryogenic amplifier for the rocket experiment, and use Hf-STJ without a grating for the future satellite experiment. The present status of the detector R\&D and the experimental design for the COBAND experiment is reported.
\end{abstract}

EPS-HEP2017, European Physical Society conference on High Energy Physics

5-12 July 2017

Venice, Italy

[1]Speaker E-mail : skim@hep.px.tsukuba.ac.jp 


\section{Introduction}

We have developed the Superconducting Tunnel Junction (STJ) detector for the cosmic background neutrino $(\mathrm{CvB})$ decay search experiment. The motivation of this experiment is to determine neutrino masses and to observe the cosmic background neutrino. We reported the proposal of rocket and satellite experiments of the cosmic background neutrino decay search which is called COBAND experiment [1]. We plan to perform a rocket experiment in 2020 in the earliest, aiming at improving the current experimental lower limit $3 \times 10^{12}$ years of the neutrino lifetime up to $10^{14}$ years by a factor of 100 in a 5-minute measurement. In the normal hierarchy case where the $v_{3}$ is the heaviest neutrino, $v_{3}$ decays into $v_{2}$ or $v_{1}$ as $v_{3} \rightarrow v_{2}\left(\right.$ or $\left.v_{1}\right)+\gamma$. Neutrino lifetime is predicted to be $10^{17}$ years by left-right symmetric model [2], and $10^{12}-10^{14}$ years by a MSSM extension model with vector-like lepton generation [3]. As the neutrino lifetime is so long as to be much larger than the age of the universe, the most promising method is to observe the decay of the cosmic background neutrino. In the $v_{3}$ rest frame, the decay photon energy $\mathrm{E}_{0}$ is related to the neutrino masses by $\mathrm{E}_{0}=\left(\mathrm{m}_{3}{ }^{2}-\mathrm{m}_{2}{ }^{2}\right) / 2 \mathrm{~m}_{3} \equiv \Delta \mathrm{m}_{32}{ }^{2} / 2 \mathrm{~m}_{3}$, where $\mathrm{m}_{3}$ and $\mathrm{m}_{2}$ are the masses of $v_{3}$ and $v_{2}$, respectively. Since $\Delta \mathrm{m}_{32^{2}}$ was measured to be $(2.45 \pm 0.05) \times 10^{-3} \mathrm{eV}^{2}$ by the oscillation experiments [4], we can determine $\mathrm{m}_{3}$ by measuring this $\mathrm{E}_{0}$. The upper limit of $\mathrm{m}_{3}$ is given as $\Sigma \mathrm{m}_{\mathrm{i}}<0.23 \mathrm{eV}$ from recent measurements of cosmic microwave background (CMB) in combination with baryon acoustic oscillation (BAO) measurements, and consequently, $\mathrm{m}_{3}$ is within the range of $50 \mathrm{meV}<\mathrm{m}_{3}<87 \mathrm{meV}$ on the assumption of the normal neutrino mass hierarchy. When we consider $\mathrm{m}_{3}$ to be $50 \mathrm{meV}$, $\mathrm{E}_{0}$ is $25 \mathrm{meV}$ in the far-infrared region. The photon energy spectrum expected from the cosmic background neutrino $(\mathrm{C} v \mathrm{~B})$ decay with the lifetime of $10^{14}$ years is shown in Fig.1. The energy cutoff at high energy end is the photon energy from the decay of a neutrino at rest. As the cosmic background neutrino is

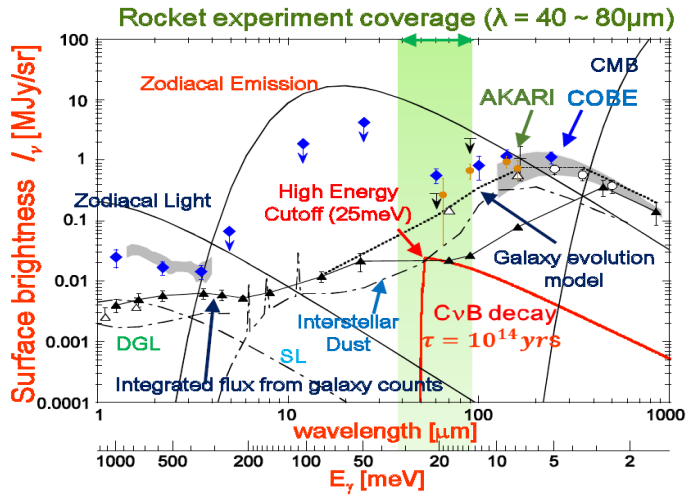

Fig.1. Photon energy spectra shown as surface brightness from the cosmic background neutrino $(C v B)$ decay with a neutrino lifetime of 1014 years. Backgrounds of the zodiacal emission and $C I B$ measured with $C O B E$ and AKARI are also shown. going far away from us by the universe expansion, it causes the low energy tail due to the red shift effect. The backgrounds against the $\mathrm{C} v \mathrm{~B}$ decay are overwhelming zodiacal emission and cosmic infrared background (CIB). By measuring the energy spectrum of the zodiacal emission with the $\mathrm{C} v \mathrm{~B}$ decay, we can see the $\mathrm{C} v \mathrm{~B}$ decay signal as a high energy cutoff. So we require the following characteristics for the detector: to measure continuously photon energy around $25 \mathrm{meV}$ with energy resolution better than $2 \%$ to identify the sharp energy cutoff, and to work at rocket or satellite experiment because a far-infrared photon from the space cannot reach us through air. As a detector of farinfrared photons with high energy resolution, we are developing the following Superconducting Tunnel Junction (STJ) photon detectors: Nb/Al-STJ with a diffraction grating and Hf-STJ without a grating. This paper reports on the development of Nb/Al-STJ and Hf-STJ. We plan to perform a rocket experiment with a STJ based detector in 2021 in the earliest [1] with 8 
spectrometers each of which consists of 50-pixels of Nb/Al-STJ and a diffraction grating, to measure the photon energy in the energy range between $15 \mathrm{meV}$ and $30 \mathrm{meV}(\lambda=40 \sim 80 \mu \mathrm{m})$, and to measure the direction dependence. The telescope diameter is $15 \mathrm{~cm}$ and the field of view is $0.006^{\circ} \times 0.05^{\circ}$. The expected rate from zodiacal background is $15 \mathrm{kHz}$ per 50 -pixel-spectrometer and $300 \mathrm{~Hz}$ per pixel. We will also search for the neutrino decay for the neutrino lifetime of 2 $\times 10^{17}$ year which is predicted by left-right symmetric model in a 100-days satellite based measurement with Hf-STJ after 2025.

\section{Development of Superconducting Tunnel Junction Detector}

\subsection{Development of Nb/AI-STJ with SOI cryogenic preamplifier}

We are developing $\mathrm{Nb} / \mathrm{Al}-\mathrm{STJ}$ single photon detectors to cope with $2 \%$ energy resolution at $\mathrm{E}_{\gamma}=25 \mathrm{meV}$ with a diffraction grating for the rocket experiment. As a basic properties of STJ detector, we measure the current-voltage (I-V) curve . From this I-V curve, we know the leakage current in the flat region $(|\mathrm{V}|<2 \Delta / \mathrm{e})$ and the energy gap $\Delta$ from the width of the flat region. Critical current is obtained from the height of Josephson current. We operate the STJ detector in the flat region after suppressing the Josephson current by applying a magnetic field parallel to the insulator layer. The fluctuation of the leakage current in this region causes a noise against the signal. We have a requirement for the leakage current of $\mathrm{Nb} / \mathrm{Al}-\mathrm{STJ}$ to be less than $0.1 \mathrm{nA}$ to detect a single far-infrared photon for the COBAND experiment. Nb/Al-STJ made at AIST CRAVITY system satisfied this requirement [1]. For seven samples of $20 \mathrm{~mm}$-square STJ's, the leakage current was $39 \pm 13 \mathrm{pA}$ at $0.3 \mathrm{mV}$ and $400 \mathrm{mK}$. The STJ has a sandwich structure of $\mathrm{Nb}(200 \mathrm{~nm}) / \mathrm{Al}(70 \mathrm{~nm}) / \mathrm{AlOx} / \mathrm{Al}(70 \mathrm{~nm}) / \mathrm{Nb}(100 \mathrm{~nm})$, of which $\Delta$ was measured to be $0.57 \mathrm{meV}$. Quasi-particles near the barrier can mediate Cooper pairs, resulting in true signal gain. This gain is called a trapping gain $\mathrm{G}_{\mathrm{Al}}$ which is about 10 for $\mathrm{Al}$. The number of quasi-particles $\mathrm{N}_{\mathrm{qp}}$ made in $\mathrm{Nb} / \mathrm{Al}$-STJ is given by $\mathrm{N}_{\mathrm{qp}}=\mathrm{G}_{\mathrm{Al}} \mathrm{E}_{\gamma} /(1.7 \Delta)$. So for $25 \mathrm{meV}$ photon, we have 250 quasiparticles. The STJ response signal to visible laser light pulse showed the response speed of a few $\mu$ sec which is fast enough for the COBAND experiment. The COABND experiment has a requirement for noise less than $30 \mathrm{e}$ after $50 \mu \mathrm{sec}$ integration. To reduce the electronics noise down to $30 \mathrm{e}$ level, we use a cryogenic amplifier which is placed close to Nb/Al-STJ. We have developed the cryogenic amplifier which works at $0.3 \mathrm{~K}$ using SOI (Silicon-On-Insulator) technique [5]. SOI-MOSFET is created on an insulator and separated from each other by the insulator and has very thin depletion layer around $50 \mathrm{~nm}$. Then we did the amplification test of the STJ signal with this SOI cryogenic amplifier. Both the $20 \mu$-square Nb/Al-STJ and the SOI amplifier were placed on the stage of ${ }^{3} \mathrm{He}$ sorption refrigerator and operated at $350 \mathrm{mK}$. We illuminated visible laser pulse light of $465 \mathrm{~nm}$ wavelength on the STJ. The STJ signal was amplified by a gain factor of 70 with the SOI cryogenic amplifier at $350 \mathrm{mK}$. We improved the signal-to-noise ratio significantly with this amplifier. Next we made the SOI cryogenic charge amplifiers with gains of $0.3 \mathrm{mV} / \mathrm{fC}, 3 \mathrm{mV} / \mathrm{fC}$, $15 \mathrm{mV} / \mathrm{fC}$ and $1.2 \mathrm{~V} / \mathrm{fC}$. About the gain of $1.2 \mathrm{~V} / \mathrm{fC}$,

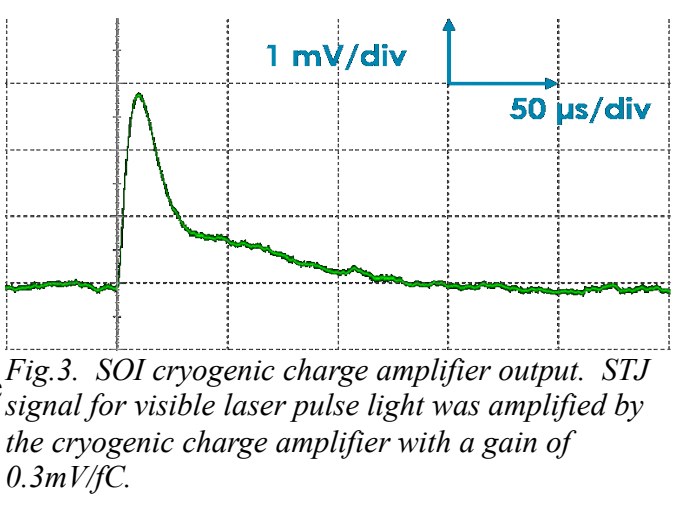


we designed the charge amplifier with voltage amplification part after charge amplification part. This charge amplifier is expected to satisfy our requirement for noise less than $30 \mathrm{e}$ after $50 \mu \mathrm{sec}$ integration. We tested the charge amplifier with a gain of $0.3 \mathrm{mV} / \mathrm{fC}$ to amplify the STJ signal and found it working as well as designed as shown in Fig.2. In this test, both the $20 \mu$-square $\mathrm{Nb} / \mathrm{Al}$-STJ and the SOI charge amplifier were placed on the stage of ${ }^{3} \mathrm{He}$ sorption refrigerator and operated at $350 \mathrm{mK}$. The test of the other charge amplifiers is undeway.

\subsection{Development of Hf-STJ}

As Hf has much smaller energy gap of $21 \mu \mathrm{eV}$ than $\mathrm{Nb}(\Delta=1.55 \mathrm{meV})$ and $\mathrm{Al}(\Delta=0.172 \mathrm{meV})$, Hf-STJ can work as a single far-infrared photon detector with $2 \%$ energy resolution. We have developed Hf-STJ and observed the STJ signal response for visible laser pulse light from $\mathrm{Hf} / \mathrm{Al}-$ STJ with a structure of $\mathrm{Hf}(250 \mathrm{~nm}) / \mathrm{Al}(10 \mathrm{~nm}) / \mathrm{HfOx}(1.5 \mathrm{~nm}) / \mathrm{Hf}(400 \mathrm{~nm})$ for the first time as reported in [6]. Our present target is to decrease the leakage current of Hf-STJ. The COBAND satellite experiment require the Hf-STJ leakage current less than $10 \mathrm{pA}$ at $50 \mathrm{mK}$. By decreasing the size of the STJ junction of $\mathrm{Hf}(250 \mathrm{~nm}) / \mathrm{HfOx}(1.5 \mathrm{~nm}) / \mathrm{Hf}(400 \mathrm{~nm})$ from $200 \mu$-square to $10 \mu$ square, we could reduce the leakage current to $200 \mathrm{nA}$ at $20 \mu \mathrm{V}$ and $150 \mathrm{mK}$ by a factor of 570 . We plan to make small-size Hf/Al-STJ's to reduce the leakage current and to measure the leakage current of all types of Hf-STJ samples at lower temperature than $50 \mathrm{mK}$.

\section{Summary}

We are developing STJ-based far-infrared photon detector to search for the cosmic background neutrino decay, COBAND experiment. Nb/Al-STJ was proved to satisfy the leakage current requirement for COBAND experiment. The visible light response signal of $\mathrm{Nb} / \mathrm{Al}-\mathrm{STJ}$ was amplified by a SOI-technology-based cryogenic amplifier placed at $350 \mathrm{mK}$ successfully for both types of voltage amplification and charge amplification. The leakage current of Hf-STJ was reduced by decreasing the junction size and now planned to be tested at lower temperature than $50 \mathrm{mK}$.

\section{Acknowledgement}

This work was supported by the Ministry of Education, Science, Sports and Culture of Japan (MEXT KAKENHI Grant Number 25105007). This work was also supported by KEK Detector Technology Project and Tomonaga Center for the History of the Universe (TCHoU) at University of Tsukuba.

\section{References}

1. S. H. Kim, K. Takemasa, Y. Takeuchi and S. Matsuura, J. Phys. Soc. Jpn., 81024101 (2012). S.H.Kim et al., Physics Procedia 37 667-674 (2012). S. H. Kim et al., JPS Conf. Proc. 1, 013127 (2014). Y. Takeuchi et al., PoS(TIPP2014)155(2014). Y. Takeuchi et al., 2015 IEEE International, 551-555 (2015).

2. M. A. B Beg, W. J. Marciano and M. Rudeman, Phys. Rev. D17, 1395 (1978).

3. A. Aboubrahim, T. Ibrahim and P. Nath, Phys. Rev. D88, 013019 (2013).

4. C. Patrignani et al. (Particle Data Group), Chin. Phys. C 40, 010001 (2016).

5. K. Kasahara et al. PoS (TIPP2014) 074 (2014). K. Kiuchi et al., FERMILAB-CONF-15-355-E-TD (2015) S. H. Kim et al. , SPPHY (TIPP2017) 213. 242 (2018).

6. K. Takemasa et al. , SPPHY (TIPP2017) 213. 254 (2018). 